\title{
Cornell potential for anisotropic QGP with non-zero chemical potential
}

\author{
Irina Aref'eva ${ }^{1, *}$, Kristina Rannu $^{2, * *}$, and Pavel Slepov ${ }^{1, * * *}$ \\ ${ }^{1}$ Steklov Mathematical Institute, Russian Academy of Sciences, Gubkina, str. 8, 119991, Moscow, \\ Russia \\ ${ }^{2}$ Peoples Friendship University of Russia, Miklukho-Maklaya str. 6, 117198, Moscow, Russia
}

\begin{abstract}
In this work we consider holographical reproduction of Cornell potential for hot and dense anisotropic quark-qluon plasma. We concentrate on the string tension gived by linear term of the Cornell potential.
\end{abstract}

\section{Introduction}

For holographical description of hot and dense anisotropic quark-qluon plasma (QGP) we use the ansatz

$$
d s^{2}=\frac{b(z)}{z^{2}}\left[-g(z) d t^{2}+d x^{2}+z^{2-\frac{2}{v}}\left(d y_{1}^{2}+d y_{2}^{2}\right)+\frac{d z^{2}}{g(z)}\right]
$$

where $b(z)=e^{\frac{c z^{2}}{2}}$ is the warp-factor, $g(z)$ is the blackening function and $v$ is the parameter of anisotropy, and the action in Einstein frame [1]

$$
\begin{aligned}
\mathcal{S}=\frac{1}{16 \pi G_{5}} \int d^{5} x \sqrt{-g}\left[R-\frac{f_{1}(\phi)}{4} F_{(1)}^{2}-\frac{f_{2}(\phi)}{4} F_{(2)}^{2}-\frac{1}{2} \partial_{\mu} \phi \partial^{\mu} \phi-V(\phi)\right], \\
F_{\mu \nu}^{(1)}=\partial_{\mu} A_{v}-\partial_{v} A_{\mu} \Rightarrow A_{\mu}^{(1)}=A_{t}(z) \delta_{\mu}^{0}, \\
F_{\mu \nu}^{(2)}=q d y^{1} \wedge d y^{2} \Rightarrow F_{23}^{(2)}=q,
\end{aligned}
$$

where $\phi=\phi(z)$ is the scalar field, $f_{1}(\phi)$ and $f_{2}(\phi)$ are the coupling functions associated with the Maxwell fields $A_{\mu}$ and $F_{\mu \nu}^{(2)}$ correspondingly, $q$ is the constant and $V(\phi)$ is the scalar field potential. We take $v=4.5$ to fit the experimental data [3,4]. For $v=1$ we get isotropic description.

\footnotetext{
*e-mail: arefeva@mi.ras.ru

**e-mail: rannu_ka@pfur.ru

***e-mail: slepov@mi.ras.ru
} 
Solutions for the blackening function, scalar field and temperature were obtained in [1]:

$$
\begin{aligned}
& g=1-\frac{z^{2+\frac{2}{v}}}{z_{h}^{2+\frac{2}{v}}} \frac{\left(\mathfrak{5}\left(\frac{3}{4} c z^{2}\right)\right.}{\mathfrak{5}\left(\frac{3}{4} c z_{h}^{2}\right)}-\frac{\mu^{2} c z^{2+\frac{2}{v}} e^{\frac{c z_{h}^{2}}{2}}\left(\mathfrak{5}\left(c z^{2}\right)\right.}{4\left(1-e^{\frac{c z_{h}^{2}}{4}}\right)^{2}}\left(1-\frac{\mathfrak{5}\left(c z_{h}^{2}\right)}{\mathfrak{5}\left(c z^{2}\right)} \frac{\mathfrak{5}\left(\frac{3}{4} c z^{2}\right)}{\mathfrak{5}\left(\frac{3}{4} c z_{h}^{2}\right)}\right), \quad \mathfrak{5}(x)=\sum_{n=0}^{\infty} \frac{(-1)^{n} x^{n}}{n !\left(1+n+\frac{1}{v}\right)}, \\
& \phi=\frac{1}{2 \sqrt{2} v}\left\{\sqrt{3 c^{2} v^{2} z^{4}-18 c v^{2} z^{2}+8(v-1)}-\sqrt{3 c^{2} v^{2} z_{h}^{4}-18 c v^{2} z_{h}^{2}+8(v-1)}+\right. \\
& +2 \sqrt{2(v-1)} \ln \left(\frac{z^{2}}{z_{h}^{2}}\right)-3 \sqrt{3} v \ln \left(\frac{\sqrt{3 c^{2} v^{2} z^{4}-18 c v^{2} z^{2}+8(v-1)}-\sqrt{3} v\left(3-c z^{2}\right)}{\sqrt{3 c^{2} v^{2} z_{h}^{4}-18 c v^{2} z_{h}^{2}+8(v-1)}-\sqrt{3} v\left(3-c z_{h}^{2}\right)}\right)- \\
& \left.-2 \sqrt{2(v-1)} \ln \left(\frac{9 c v^{2} z^{2}-8(v-1)-\sqrt{2(v-1)} \sqrt{3 c^{2} v^{2} z^{4}-18 c v^{2} z^{2}+8(v-1)}}{9 c v^{2} z_{h}^{2}-8(v-1)-\sqrt{2(v-1)} \sqrt{3 c^{2} v^{2} z_{h}^{4}-18 c v^{2} z_{h}^{2}+8(v-1)}}\right)\right\}, \\
& T=\frac{g^{\prime}\left(z_{h}\right)}{4 \pi}=\frac{e^{-\frac{3 c z_{h}^{2}}{4}}}{2 \pi z_{h}}\left|\frac{1}{\mathfrak{5}\left(\frac{3}{4} c z_{h}^{2}\right)}+\frac{\mu^{2} c z_{h}^{2+\frac{2}{v}} e^{\frac{c z_{h}^{2}}{4}}}{4\left(1-e^{\frac{c z_{h}^{2}}{4}}\right)^{2}}\left(1-e^{\frac{c z_{h}^{2}}{4}} \frac{\left(\mathfrak{5}\left(c z_{h}^{2}\right)\right.}{\mathfrak{5}\left(\frac{3}{4} c z_{h}^{2}\right)}\right)\right| .
\end{aligned}
$$

The corresponding diagram for confinement/deconfinement phase transition is depicted on Fig.1. Orange and cyan curves show the Hawking-Page-like transition (background) lines in isotropic and anisotropic cases. Green curve corresponds to the phase transition line, determined by the Wilson loop in isotropic case. Anisotropy leads to different Wilson lines for the strings, oriented along the longitudinal (blue) and transversal (magenta) directions. Properties of this phase diagram is discussed in more detailes in $[1,2]$. You can also find more informa-

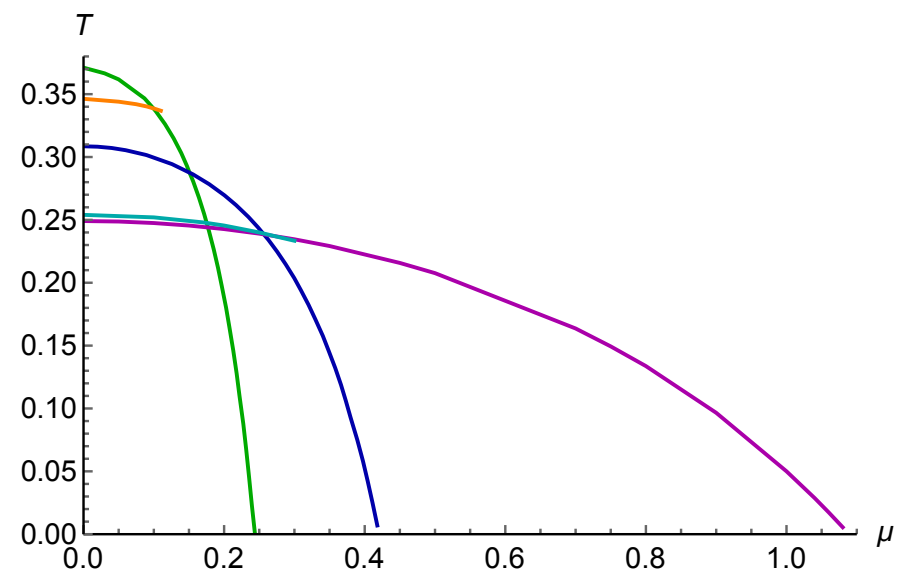

Figure 1. Confinement-deconfinement phase transitions of Wilson lines for $v=1$ (green), longitudinal and transversal components for $v=4.5$ (dark blue and dark magenta); the background transition lines for $v=1$ (orange) and $v=4.5$ (dark cyan). 
tion on motivation and reasoning of the holographic approach in talk by I. Ya. Aref'eva from this conference [4].

\section{Wilson loop}

According to the holographic approach [5-7] the expectation value of the temporal Wilson loop is

$$
W\left[C_{\vartheta}\right]=e^{-S_{\vartheta, t}}
$$

oriented along vector $\vec{n}$, such that $n_{x}=\cos \vartheta, n_{y}=\sin \vartheta$. The world sheet presented in Fig.2 is parameterized as

$$
X^{0} \equiv t, \quad X^{1} \equiv x=\xi \cos \vartheta, \quad X^{2} \equiv y_{1}=\xi \sin \vartheta, \quad X^{3} \equiv y_{2}=\text { const }, \quad X^{4} \equiv z=z(\xi) .
$$

Within our solution the Nambu-Goto action for the test string is [2]

$$
S=-\frac{\tau}{2 \pi \alpha^{\prime}} \int d \xi \frac{b(z(\xi))}{z(\xi)^{2}} e^{\sqrt{\frac{2}{3}} \phi(z)} \sqrt{g(z(\xi))\left(\cos ^{2}(\theta)+z^{2-\frac{2}{v}} \sin ^{2}(\theta)\right)}, \quad \tau=\int d t,
$$

where $\theta$ is the angle between quarks line and heavy-ion collisions line.

The string tension can be calculated as

$$
\sigma_{D W}=\frac{b_{s}\left(z_{D W}\right)}{z_{D W}^{2}} \sqrt{g\left(z_{D W}\right)\left(\cos ^{2}(\theta)+z_{D W}^{2-\frac{2}{v}} \sin ^{2}(\theta)\right)}
$$

where we introduced string warp-factor $b_{s}(z)=b(z) e^{\sqrt{\frac{2}{3}} \phi(z)}$. Taking angles $\theta=0$ and $\theta=\pi / 2$, we have particular expressions for tension of longitudinal and transversal strings:

$$
\sigma_{D W x}=\frac{b_{s}\left(z_{D W}\right)}{z_{D W}^{2}} \sqrt{g\left(z_{D W}\right)}, \quad \sigma_{D W y}=\frac{b_{s}\left(z_{D W}\right)}{z_{D W}^{1+\frac{1}{v}}} \sqrt{g\left(z_{D W}\right)}
$$

The domain wall position $z_{D W}$ is determined by the condition $\partial \sigma / \partial z=0$ and obviousely depends on quark pair orientation [2]. For other aspects of the Wilson loop consideration within this model see talk by P. Slepov from this conference [8].

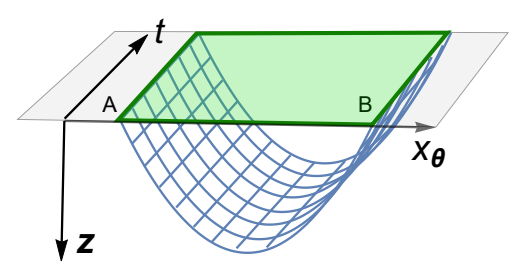

A

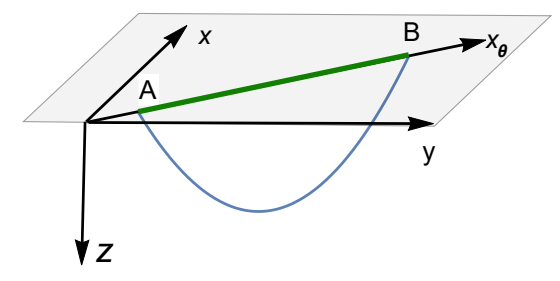

Figure 2. A. Wilson loop and the world sheet. B. Projection of the world sheet to fixed $t$. 

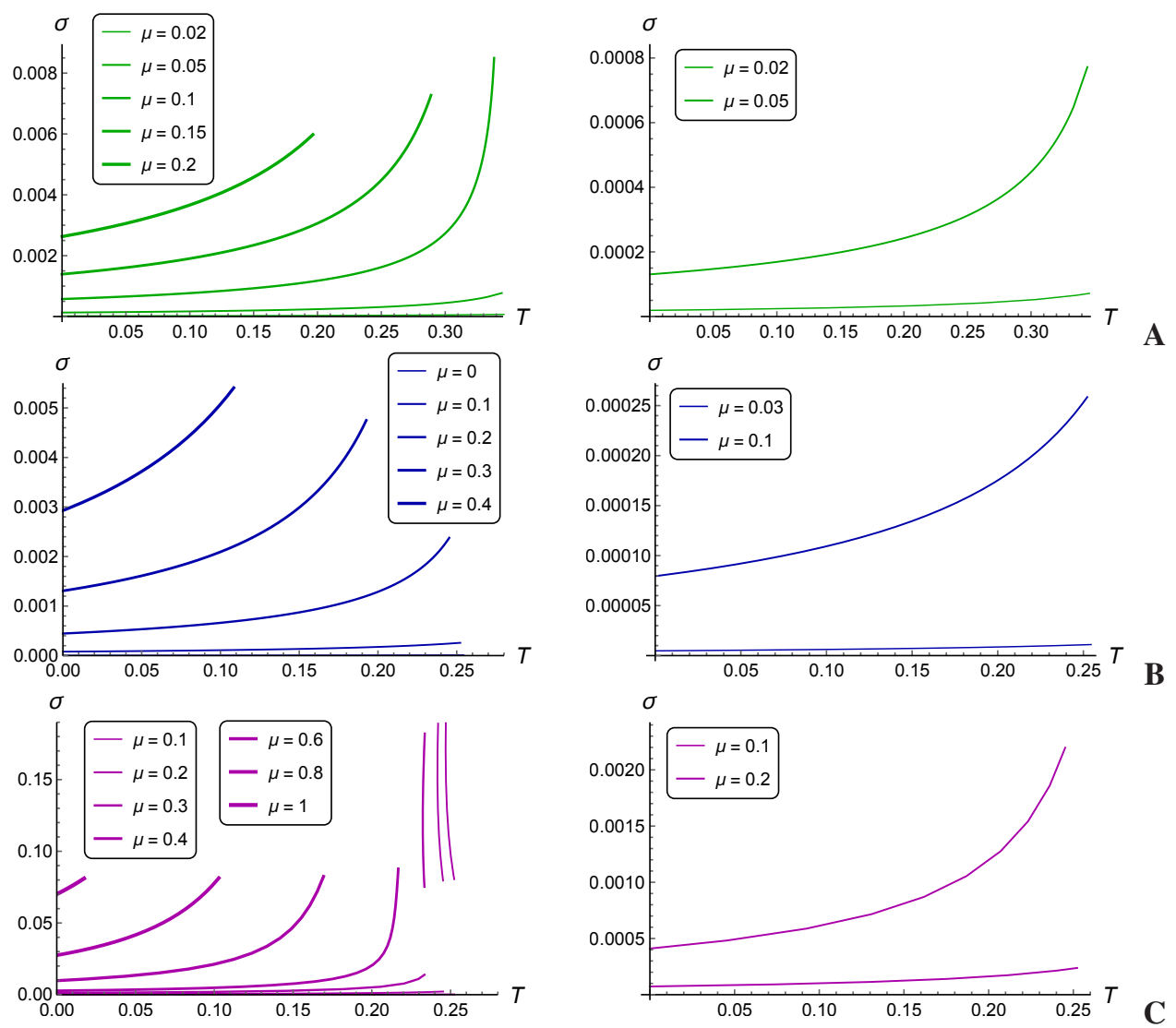

Figure 3. String tension as function of temperature for $v=1, \mu=0,0.05,0.1,0.15,0.2$ (A) and $v=4.5$, longitudinal for $\mu=0,0.1,0.2,0.3,0.4$ (B) and transversal for $\mu=0,0.2,0.4,0.6,0.8,1$ (C).

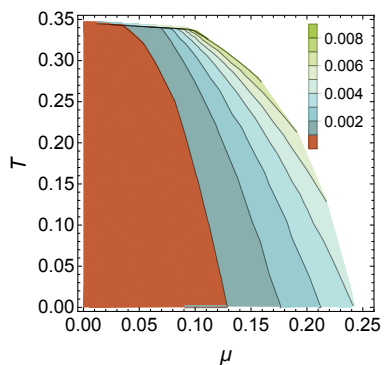

A

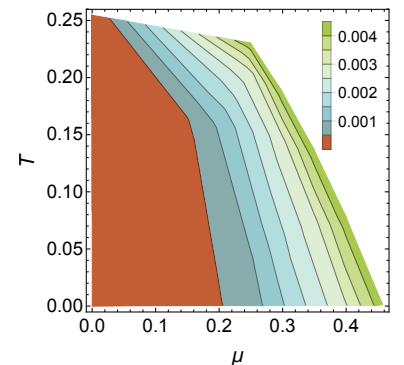

B

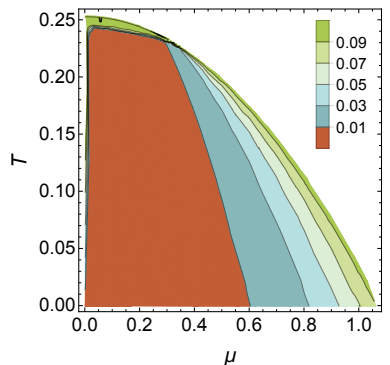

$\mathrm{C}$

Figure 4. Contour plot of the string tension as function of chemical potential $\mu$ and temperature $T$ for $v=1$ (A) and $v=4.5$, longitudinal (B) and transversal (C).

The string tension turns out to increase with temperature and chemical potential. On Fig.3 $\sigma(T)$ curves, corresponding to different values of chemical potential are shown. The avaliable temperatures are limited by the Hawking-Page-like phase transition line. 
The pictures for isotropic case (Fig.3A) and the longitudinal orientation of the string in anisotropic case (Fig.3B) look similar, but anisotropic curves have lesser temperatures, according to the phase transition diagram (Fig.1), and lesser string tension values.

The main interest is the behavior for the transversal orientation of the string in anisotropic case (Fig.3C). For small chemical potentials $\sigma(T)$ becomes a multivalued function near the Hawking-Page-like temperatures. This is caused by the narrow instability region between the transversal Wilson line and the Hawking-Page-like line (Fig.1). Curves for $\mu=0.1,0.2,0.3$ on (Fig.3C could be continuous, but they were splited into two parts by the temperature cut-off due to Hawking-Page-like phase transition. Note, that this cut-off occurs at smaller temperature for larger chemical potential. Such a behavior for isotropic and longitudinal anisotropic cases is not be seen, as should be located in the deconfinement area, where we cannot talk about the string tension.

Fig.4 displays a continious "height map" of string tension $\sigma(\mu, T)$.

\section{Cornell potential}

In our model representations for the character length of the string and the action have the form:

$$
\begin{gathered}
\ell=2 \int_{0}^{z_{*}} \frac{b_{s}\left(z_{*}\right)}{b_{s}(z)} \frac{z^{2}}{z_{*}^{2}} \frac{\sqrt{g\left(z_{*}\right)\left(\cos ^{2}(\theta)+z_{*}^{2-\frac{2}{v}} \sin ^{2}(\theta)\right)}}{g(z)\left(\cos ^{2}(\theta)+z^{2-\frac{2}{v}} \sin ^{2}(\theta)\right)} \frac{d z}{\sqrt{1-\frac{b_{s}^{2}\left(z_{*}\right)}{b_{s}^{2}(z)} \frac{z^{4}}{z_{*}^{4}} \frac{g\left(z_{*}\right)}{g(z)} \frac{\cos ^{2}(\theta)+z_{*}^{2-\frac{2}{v}} \sin ^{2}(\theta)}{\cos ^{2}(\theta)+z^{2-\frac{2}{v}} \sin ^{2}(\theta)}}} \\
S=2 \int_{0}^{z_{*}} \frac{b_{s}(z)}{z^{2}} \frac{d z}{\sqrt{1-\frac{b_{s}^{2}\left(z_{*}\right)}{b_{s}^{2}(z)} \frac{z^{4}}{z_{*}^{4}} \frac{g\left(z_{*}\right)}{g(z)} \frac{\cos ^{2}(\theta)+z_{*}^{2-\frac{2}{v}} \sin ^{2}(\theta)}{\cos ^{2}(\theta)+z^{2-\frac{2}{v}} \sin ^{2}(\theta)}}}
\end{gathered}
$$

where $z_{*}$ is a top point. Taking angles $\theta=0$ and $\theta=\pi / 2$, we have particular expressions for length and action of longitudinal and transversal strings:

$$
\begin{aligned}
& \ell_{x}=2 \int_{0}^{z_{*}} \frac{b_{s}\left(z_{*}\right)}{b_{s}(z)} \frac{z^{2}}{z_{*}^{2}} \frac{\sqrt{g\left(z_{*}\right)}}{g(z)} \frac{d z}{\sqrt{1-\frac{b_{s}^{2}\left(z_{*}\right)}{b_{s}^{2}(z)} \frac{z^{4}}{z_{*}^{4}} \frac{g\left(z_{*}\right)}{g(z)}}}, \\
& S_{x}=2 \int_{0}^{z_{*}} \frac{b_{s}(z)}{z^{2}} \frac{d z}{\sqrt{1-\frac{b_{s}^{2}\left(z_{*}\right)}{b_{s}^{2}(z)} \frac{z^{4}}{z_{*}^{4}} \frac{g\left(z_{*}\right)}{g(z)}}} \\
& \ell_{y}=2 \int_{0}^{z_{*}} \frac{b_{s}\left(z_{*}\right)}{b_{s}(z)}\left(\frac{z^{2}}{z_{*}}\right)^{\frac{1}{v}} \frac{1}{z_{*}} \frac{\sqrt{g\left(z_{*}\right)}}{g(z)} \frac{d z}{\sqrt{1-\frac{b_{s}^{2}\left(z_{*}\right)}{b_{s}^{2}(z)} \frac{z^{2+\frac{2}{v}}}{z_{*}^{2+\frac{2}{v}}} \frac{g\left(z_{*}\right)}{g(z)}}}, \\
& S_{y}=2 \int_{0}^{z_{*}} \frac{b_{s}(z)}{z^{2}} \frac{d z}{\sqrt{1-\frac{b_{s}^{2}\left(z_{*}\right)}{b_{s}^{2}(z)} \frac{z^{2+\frac{2}{v}}}{z_{*}^{2+\frac{2}{v}}} \frac{g\left(z_{*}\right)}{g(z)}}}
\end{aligned}
$$




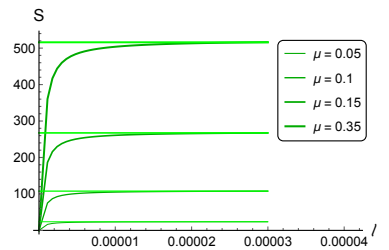

A

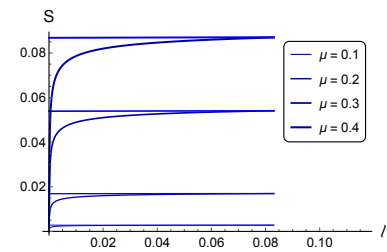

B

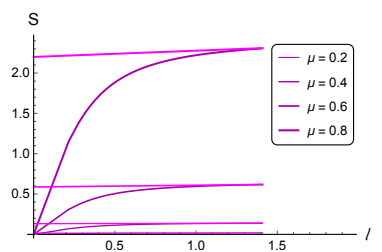

$\mathrm{C}$

Figure 5. String tension as function of chemical potential $\mu$ and temperature $T$ for $v=1$ (A) and $v=4.5$, longitudinal (B) and transversal (C) for $T=0.1$.

The numerical results for Cornell potential are shown on Fig.5. Darker curves depict the Cornell potential intself and the lighter strict lines show the incline of its linear term, determined by the corresponding string tension. To make these results for isotropic (Fig.5A), anisotropic longitudinal (Fig.5B) and transversal (Fig.5C) cases comparable we choose the temperature $T=0.1$, that is a permissible value for all three cases. Both the inclination and the action $S$ have larger values for larger chemical potential, that agrees with the results from Section 2.

\section{Acknowledgments}

This paper is based on a talk at the XXIV International Workshop High Energy Physics and Quantum Field Theory at Sochi, Russia on 22 - 29 September 2019. I would like to thank the organizers of QFTHEP'2019 for the invitation. This work was supported by Russian Foundation for Basic Research (RFBR) grant No. 18-02-40069 mega.

\section{References}

[1] I. Ya. Aref'eva and K. A. Rannu, JHEP 05, 206 (2018).

[2] I. Ya. Aref'eva, K. A. Rannu and P. S. Slepov, PLB 792, 470 (2019).

[3] I. Ya. Aref'eva and A. A. Golubtsova, JHEP 1504, 011 (2015).

[4] I. Aref'eva, “Theoretical Studies of Heavy Ion Collisions via Holography”, these proceedings.

[5] J. M. Maldacena, Phys. Rev. Lett. 80, 4859 (1998).

[6] S. J. Rey, S. Theisen and J. T. Yee, Nucl. Phys. B 527, 171 (1998).

[7] A. Brandhuber, N. Itzhaki, J. Sonnenschein and S. Yankielowicz, Phys. Lett. B 434, 36 (1998).

[8] I. Aref'eva, A. Patrushev and P. Slepov, "Holographic Entanglement Entropy in Anisotropic Background with Confinement-Deconfinement Phase Transition" (in preparation); P. Slepov, "Entanglement entropy in strongly correlated systems with confinement/deconfinement phase transition and anisotropy", these proceedings. 